\title{
Granisetron Transdermal Patch for Treatment of Nausea in Patients Undergoing Cisplatin Based Chemotherapy for Cervical Cancer
}

\author{
Helen Dunnington ${ }^{1}$, Rodney Hunter ${ }^{2}$, Michael Frumovitz ${ }^{3}$, Judith Smith ${ }^{1,2,3^{*}}$ \\ ${ }^{1}$ University of Texas Medical School at Houston, Houston, USA; ${ }^{2}$ College of Pharmacy, Texas Southern University, Houston, USA; \\ ${ }^{3}$ University of Texas M. D. Anderson Cancer Center, Houston, USA. \\ Email: *helen.a.dunnington@uth.tmc.edu
}

Received September $10^{\text {th }}, 2012$; revised October $13^{\text {th }}, 2012$; accepted October $24^{\text {th }}, 2012$

\begin{abstract}
Objective: The purpose of this study was to define the incidence of chemotherapy-induced nausea and vomiting (CINV) in newly diagnosed cervical cancer patients receiving cisplatin and radiation treatment; and to determine if the granisetron transdermal patch (Sancuso ${ }^{\circledR}$ ) would be appropriate to recommend as part of standard antiemetic regimen for the cisplatin radiation chemotherapy order set. Methods: This is a retrospective case-controlled study of cervical cancer patients receiving cisplatin chemotherapy with radiation (cisXRT); comparing patients receiving the granisetron transdermal patch to matched patients receiving oral $5 \mathrm{HT}_{3}$ blockers. All patients prescribed cisXRT between September 15th 2008 and November 30, 2011 were identified using pharmacy dispensing records. Patients were included if they received at least a partial dose of cisXRT and Sancuso ${ }^{\circledR}$ patch as standard antiemetic prophylaxis prior to cisXRT for cervical cancer treatment. Exclusion criteria included concomitant investigational agents, biotherapy and/or chemotherapy agents; prior chemotherapy; or incomplete or restricted medical records. Patients will be matched based on age. Patients were matched 3:1 (oral:patch). A total of 404 patients that received and completed cisXRT were identified utilizing an existing de-identified database from previous study were reviewed to evaluate parameters of interest. Results: A total of 285 patients' medication records were reviewed for Sancuso ${ }^{\circledR}$ use, and 47 were identified. Of these 47 charts only five patient cases met eligibility criteria to be included in the study. All five patients that received the granisetron patch had at least three known risk factors for nausea. The nausea/vomiting in these patients did not worsen after receiving the Sancuso ${ }^{\circledR}$ patch, and four out of five had subjective improvement. CINV was unrelated to changes in laboratory values or incidence of other toxicity and was not dose-related. Conclusions: While no definitive conclusions could be drawn from this retrospective analysis, the limited data suggests that patients' nausea and vomiting did not worsen after receiving the Sancuso ${ }^{\circledR}$ patch, and four out of five patients had subjective improvement. The challenges met and limitations identified justify the need for a prospective study that is now underway to control other contributing variables and evaluate overall efficacy of the granisetron patch for controlling CINV in patients receiving cisplatin plus radiation.
\end{abstract}

Keywords: Granisetron; Nausea; Vomiting; Cisplatin; Radiation

\section{Introduction}

With current screening tools, the incidence of cervical cancer is decreasing in the United States. In 2012 there will be an estimated 12,170 new cases of and 4220 deaths in the United States from cervical cancer [1]. Treatment typically consists of either primary surgery or primary radiation combined with chemotherapy. Peters and colleagues studied the addition of cisplatin-based chemotherapy to radiation therapy compared to radiation therapy alone in postoperative patients with early stage cancer [2]. This study demonstrated a decrease in recur-

"Corresponding author. rence and improvements in progression-free and overall survival when chemotherapy was added to pelvic radiation therapy [2]. This demonstrated improvement came with the addition of greater toxicity.

Multiple studies have investigated acute toxicities associated with concurrent cisplatin-based chemotherapy and radiation therapy [3,4]. In a review of 19 randomized controlled trials of combined chemoradiation in cervical cancer, Kirwan and colleagues studied the additional acute and late toxicities. Studies using cisplatin plus radiation were analyzed separately from studies using radiation alone. When compared to the combined studies, the cisplatin trials had similar grade 1 and 2 tox- 
icities with all showing increased hematologic toxicities compared to the control studies. Grade 3 and 4 acute toxicities for cisplatin were also similar; the predominant toxicities were gastrointestinal and hematologic. While the cisplatin studies showed a significant increase in grade 3 and 4 anemia, the combined studies did not. The authors concluded that the additional toxicities were acceptable given the survival benefit of chemoradiation [3].

Although the treatment toxicities are considered acceptable from a survival standpoint, patient compliance is imperative to successful therapy. In a Polish study, Serkies and Jassem investigated patient compliance and acute toxicity in 112 patients who were scheduled to receive weekly cisplatin and radiotherapy for cervical cancer management. The chemotherapy treatment schedule included five cycles of cisplatin. Overall, $74 \%$ of patients received at least four cycles of chemotherapy. The five cycles scheduled were not administered because of treatment toxicity in 35 patients (31\%). Another 23 patients (21\%) did not receive the full five cycles because of noncompliance with the treatment schedule. Of the multiple toxicities, gastrointestinal effects were seen in 42 patients; intense vomiting was seen in six patients; and three discontinued cisplatin as a result of worsening performance status [4]. To improve treatment compliance, toxicity needs to be addressed and treated.

Due to the highly emetic nature of cisplatin, prophylactic antiemetics are essential. Nausea, with or without vomiting, occurs in approximately $70 \%-80 \%$ of patients receiving chemotherapy; $10 \%-44 \%$ of patients suffer from anticipatory nausea, vomiting, or both. Females, younger patients ( $<50$ years old), and patients with a history of nausea and vomiting or motion sickness will be more likely to develop chemotherapy induced nausea and vomiting (CINV). Also, patients with high tumor burden, or opioid use for pain management will have increased risk for CINV. In contrast, patients with a history of chronic alcoholism are less likely to develop CINV [5]. Nausea and vomiting can affect functional ability and quality of life, and can even result in malnutrition and electrolyte imbalances. Current recommendations to prevent acute emesis specify a three-drug regimen using a 5- $\mathrm{HT}_{3}$ receptor antagonist, dexamethasone, and aprepitant. For delayed emesis, a two-drug regimen is recommended using aprepitant with dexamethasone [6]. Despite these therapies, compliance problems continue among patients who take oral medications and suffer from xerostomia or anticipatory nausea [7]. A newer option that may improve treatment compliance in cervical cancer patients receiving cisplatin-based chemotherapy is the granisetron transdermal patch.

On September 12, 2008, the FDA approved the granisetron transdermal patch $\left(\right.$ Sancuso $^{\circledR}$ ). It is the first sero- tonin $5-\mathrm{HT}_{3}$ receptor antagonist available in a transdermal form indicated for the prevention of CINV. The patch $(3.1 \mathrm{mg} / 24 \mathrm{hr})$ reaches Cmax at approximately 48 hours after application and may be worn up to seven days. It is recommended that the patch be applied to the upper arm at least 24 hours prior to the start of chemotherapy and left in place for a minimum of 24 hours after completion of chemotherapy $[8,9]$. It provides low peak plasma concentrations $(5.4 \mathrm{ng} / \mathrm{mL})$ and sustained levels within the blood for approximately five days before gradually decreasing. Levels are maintained similar to that obtained with $2 \mathrm{mg}$ of oral granisetron administered every day over a period of six days [7]. Transdermal drug delivery, compared to oral administration, offers less of a burden to patients suffering from xerostomia or anticipatory nausea while avoiding first pass metabolism and giving more consistent drug levels over the course of therapy. These advantages can be met with I.V. administration, but the patch is less invasive and can be managed reliably in an outpatient setting [5]. Previous studies have shown equivalence in clinical efficacy among the $5 \mathrm{HT}_{3}$ antagonists as well as equivalence between oral and transdermal granisetron [5,7]. For patients having trouble adhering to an antiemetic drug schedule, the granisetron patch is ideal for increasing patient compliance during weekly chemoradiation with cisplatin for cervical cancer treatment.

The purpose of this study was to define the incidence of chemotherapy-induced nausea/vomiting in newly diagnosed cervical cancer patients receiving cisplatin and radiation treatment and to determine if the use of granisetron transdermal patch (Sancuso ${ }^{\circledR}$ ) would be appropriate to recommend as part of the standard antiemetic regimen for the cisplatin radiation chemotherapy order set. Secondary objectives were to observe if there have been any treatment delays or dose reductions due to CINV and if rescue antiemetics were received in those patients.

\section{Methods}

\subsection{Study Design}

This was a retrospective case-controlled study of cervical cancer patients receiving cisplatin chemoradiation (cisXRT), comparing patients receiving the granisetron transdermal patch to matched patients receiving traditional oral $5 \mathrm{HT}_{3}$ blockers. All patients prescribed cisXRT between September 15, 2008 and November 30, 2011 were identified using pharmacy dispensing records. Patients were matched 3:1 (oral:patch). The study protocol was approved by The University of Texas M.D. Anderson Cancer Center (UTMDACC) institutional review board and was exempt from informed consent because no patient identifying 
information was collected and patient anonymity was protected.

\subsection{Patient Selection and Data Collection}

Patients receiving combination CisXRT were identified through the UTMDACC Division of Pharmacy dispensing records and considered for inclusion. Criteria for inclusion were patients with a new diagnosis of cervical cancer and completion of weekly chemoradiation with cisplatin at UTMDACC. Patients were excluded if they did not have cervical cancer, were current participants of an investigational drug study, had incomplete medical records, received previous chemotherapy agents, or received other chemotherapy agents while being treated with weekly cisplatin in combination with radiation.

A historical database of 402 patients that received and completed cisplatin and radiation was evaluated for case controls. The database consisted of information collected from November 2003 to August 2006 and September 2007 to August 2008. To identify patients who received the granisetron transdermal patch, all patients prescribed cisplatin XRT between September 15, 2008 and November 30, 2011 were identified using pharmacy dispensing records.

Parameters evaluated included age, documented comorbidities, documented concomitant medications before first cycle, cisplatin chemotherapy history, baseline chemistry before each cycle of cisplatin administration (Na, Bun, $\mathrm{Cr}, \mathrm{K}, \mathrm{Mg}$ ), CBC (Hg, Plt, WBC, and ANC) at baseline and prior to each cycle of cisplatin, number of episodes of CINV that occur during treatment, treatment interventions for CINV beyond standard treatment (i.e. rescue antiemetics), complications of CINV if present, any documented side effects, documented treatment delays due to CINV and documented cisplatin dose reduction due to CINV.

Cisplatin chemotherapy was administered on a weekly basis for five weeks. Dosing was $40 \mathrm{mg} / \mathrm{m}^{2}$ with a maximum total dose of $70 \mathrm{mg}$ in $1 / 2$ normal saline with mannitol over one hour. Patients were pre-hydrated with 500 $\mathrm{mL}$ normal saline and magnesium sulfate over one hour, and post-hydrated with $500 \mathrm{~mL} \mathrm{1/2}$ normal saline with potassium chloride and magnesium sulfate over two hours. Standard antiemetics were either: oral dose of Zofran $8 \mathrm{mg} 30$ minutes prior to chemotherapy, then every eight hours for five days with dexamethasone on day 1 only, or transdermal dose of Sancuso ${ }^{\circledR}$ patch (3.1 $\mathrm{mg} / 24 \mathrm{hr}$ ) applied 24 to 48 hours prior to start of chemotherapy and worn continuously for seven days with dexamethasone on day 1 only. Rescue antiemetics were defined as any additional antiemetics administered after day 1 , beyond standard antiemetic treatment, as described, for CINV correlated to cisXRT. This would in- clude medications such as aprepitant, prochlorperazine, $\mathrm{ABH}$, metoclopramide, etc.

\subsection{Analysis}

This study was designed to evaluate current practice and identify opportunities for quality improvement of CINV in cervical cancer patients undergoing cisXRT. The proportion of patients who received rescue antiemetics was calculated from of those patients who experienced CINV. Risk factors for CINV were identified and compared between groups. Clinic notes were reviewed for documentation of worsening or improvement in nausea and vomiting after receiving Sacusco ${ }^{\circledR}$.

\section{Results}

A total of 285 patient medication records were reviewed for Sancuso ${ }^{\circledR}$ use, and 47 were identified. Of these 47 patients only five met eligibility criteria to be included in the study. Patients were excluded for reasons including: having other gynecologic malignancies, receiving previous chemotherapy, or receiving additional chemotherapeutic agents with cisplatin. Fifteen patients served as matched control patients that were pulled from the cisplatin-radiation database of 402 patients. Each study patient was matched to three control patients based on age. Baseline patient demographic and medical characteristics in these women were comparable between cases and controls (see Table 1).

In this study, twelve out of the total 20 patients (60\%) experienced CINV. All five of the granisetron patients experienced CINV. Of previously identified risk factors for CINV, the most common ones identified in this study included extremes of age (3/5) and no chronic alcohol use (2/5); other risk factors included anxiety, GERD, immune disorders, and advanced disease. Seven of the fifteen controls experienced CINV with the most common being extremes of age (5/7), advanced disease (3/7), and no history of chronic alcohol use (2/7). Female gender is a known risk factor for CINV, and therefore was present in all study patients.

Rescue antiemetics were required for CINV in all five of the Sancuso ${ }^{\circledast}$ group patients, with an average of 3.2 medications per patient, the most required being five. An average of 1.3 rescue medications per person was used for the control group, however of those with CINV there was an average of 2.3 rescue medications, the most required being four.

Treatment delays due to CINV were seen in 2/5 Sancuso $^{\circledR}$ group patients; however, none resulted in dose reductions. None of the control patients had treatment delays due to CINV; one patient had an ER visit but did not require admission and was discharged home. On 
Table 1. Demographics and results.

\begin{tabular}{|c|c|c|c|c|c|}
\hline & & Sancuso $^{\circledR}$ & $\mathrm{N}=5$ & Control & $\mathrm{N}=15$ \\
\hline Age & & $45.4(27-66)$ & & $46.5(28-67)$ & \\
\hline \multirow[t]{3}{*}{ Race } & Black & 1 & $20 \%$ & 1 & $6.67 \%$ \\
\hline & White & 4 & $80 \%$ & 11 & $73.33 \%$ \\
\hline & Hispanic & 0 & $0 \%$ & 3 & $20.00 \%$ \\
\hline CINV & & 5 & $100 \%$ & 7 & $46.67 \%$ \\
\hline \multirow[t]{9}{*}{ Risk factors for CINV } & Age & 3 & $60 \%$ & 5 & $71.43 \%$ \\
\hline & Gender & 5 & $100 \%$ & 7 & $100.00 \%$ \\
\hline & No ETOH & 2 & $40 \%$ & 2 & $28.57 \%$ \\
\hline & Advanced disease & 1 & $20 \%$ & 3 & $42.86 \%$ \\
\hline & Immune disorder & 1 & $20 \%$ & 0 & $0.00 \%$ \\
\hline & Anxiety & 1 & $20 \%$ & 0 & $0.00 \%$ \\
\hline & Underweight & 1 & $20 \%$ & 0 & $0.00 \%$ \\
\hline & GERD & 1 & $20 \%$ & 0 & $0.00 \%$ \\
\hline & Baseline NV & 1 & $20 \%$ & 0 & $0.00 \%$ \\
\hline Number of rescue medications & & 3.2 & $(2-5)$ & 2.3 & $(1-4)$ \\
\hline Treatment delay & & 2 & $40 \%$ & 0 & $0 \%$ \\
\hline Dose reduction & & 0 & $0 \%$ & 0 & $0 \%$ \\
\hline CINV worse after Sancuso ${ }^{\circledR}$ ? & & 0 & $0 \%$ & N/A & N/A \\
\hline CINV improved after Sancuso ${ }^{\circledR}$ ? & & 4 & $80 \%$ & N/A & N/A \\
\hline
\end{tabular}

average, electrolytes (sodium, potassium and magnesium) were within normal limits throughout chemotherapy for both the Sancuso ${ }^{\circledR}$ and control groups based on normal ranges from the UTMDACC laboratory.

\section{Discussion}

While cisXRT has been shown to be a more effective treatment than radiation alone, additional toxicities are associated. Nausea and vomiting are some of the most significant, often causing treatment delays, and can affect functional ability and quality of life, result in malnutrition, and electrolyte imbalances. Risk factors for chemotherapy induced nausea and vomiting include female gender, age, advanced disease, history of nausea or vomiting, and motion sickness, while alcohol is protective. Current recommendations include use of an oral $5 \mathrm{HT}_{3}$ receptor antagonist. This regimen is not adequate for all patients, and an alternative option is needed.

This analysis provides some data on the efficacy of the granisetron transdermal patch for treating CINV specific to cervical cancer patients receiving cisXRT. Of the twenty charts reviewed, 5 Sancuso ${ }^{\circledR}$ patients, and 15 controls, there was a $60 \%$ rate of CINV. This demonstrates a need for effective antiemetics in the treatment of cervical cancer with cisplatin plus radiation.

The major limitation of this study is its low power. This is partly due to the fact that the prescription for the granisetron transdermal patch is an outpatient prescrip tion, making it hard to identify. Records are not available for patients who filled their prescriptions at pharmacies outside the MD Anderson system. Also, not all dictated clinic notes or medication reconciliations specify what antiemetic medications a patient is prescribed or taking. Another reason for the low number of cases is related to the specific focus of our question; some patients were excluded due to being on other trials at the time, having previously received cisplatin, or having another gynecologic malignancy besides cervical cancer.

While this study was too small and limited to conclude whether or not the granisetron patch should be included in the cervical cancer chemotherapy order set, it does 
lend support to the need for a prospective study. From the five patients identified, none had worsening nausea and vomiting, and four out of five had improved symptoms. A prospective study would allow for greater power and less confounding to show what we anticipate to be equal efficacy of the patch to standard $5 \mathrm{HT}_{3}$ blocker antiemetics.

\section{REFERENCES}

[1] National Cancer Institute, “Cervical Cancer,” National Cancer Institute, Frederick, 2012. http://www.cancer.gov/cancertopics/types/cervical

[2] W. A. Peters, P. Y. Liu, R. J. Barrett, et al., "Concurrent Chemotherapy and Pelvic Radiation Therapy Compared with Pelvic Radiation Therapy Alone as Adjuvant Therapy after Radical Surgery in High-Risk Early-Stage Cancer of the Cervix,” Journal of Clinical Oncology, Vol. 18, No. 8, 2000, pp. 1606-1613.

[3] J. M. Kirwan, P. Symonds, J. A. Green, et al., "A Systematic Review of Acute and Late Toxicity of Concomitant Chemoradiation for Cervical Cancer," Radiotherapy and Oncology, Vol. 68, No. 3, 2003, pp. 217-226. doi:10.1016/S0167-8140(03)00197-X

[4] K. Serkies and J. Jassem, “Concurrent Weekly Cisplatin and Radiotherapy in Routine Management of Cervical
Cancer: A Report on Patient Compliance and Acute Toxicity,” International Journal of Radiation Oncology Biology Physics, Vol. 60, No. 3, 2004, pp. 814-821. doi:10.1016/j.ijrobp.2004.04.042

[5] L. Fala, "Advances in Treatment Options and Management of Chemotherapy-Induced Nausea and Vomiting," A Supplement to the Oncology Pharmacist, Vol. 3, No. 2, 2010, pp. 1-12.

[6] A. Naeim, S. M. Dy, et al., "Evidence-Based Recommendations for Cancer Nausea and Vomiting," Journal of Clinical Oncology, Vol. 26, No. 23, 2008, pp. 3903-3910. doi:10.1200/JCO.2007.15.9533

[7] J. Howell, J. Smeets, et al., "Pharmacokinetics of a Granisetron Transdermal System for the Treatment of Chemotherapy-Induced Nausea and Vomiting," Journal of Oncology Practice, Vol. 15, No. 4, 2009, pp. 223-231.

[8] Granisetron Transdermal System (Sancuso-Prostraken), “Package Insert," 2010. http://health.utah.gov/medicaid/pharmacy/ptcommittee/fil es/Criteria\%20Review\%20Documents/Antiemetics\%20(F ebrary\%202010)/Medicaid_granisetron_transderm_02031 $0 . p d f$

[9] S. Grunberg, R. A. Clark-Snow and J. Koeller, "Chemotherapy-Induced Nausea and Vomiting: Contemporary Approaches to Optimal Management," Supportive Care in Cancer, Vol. 18, No. 1, 2010, pp. S1-S10. doi:10.1007/s00520-009-0807-z

\author{
Abbreviations \\ CINV: chemotherapy induced nausea and vomiting; \\ cisXRT: cisplatin chemotherapy with radiation; \\ UTMDACC: University of Texas MD Anderson Cancer \\ Center; \\ ABH: Ativan, Benadryl, \& Haldol.
}

